\title{
Nearly optimal meshes in subanalytic sets
}

\author{
Wiesław Pleśniak
}

Received: 12 December 2011 / Accepted: 12 April 2012 /

Published online: 26 April 2012

(C) The Author(s) 2012. This article is published with open access at Springerlink.com

\begin{abstract}
We prove that any fat, subanalytic compact subset of $\mathbb{R}^{N}$ possesses a nearly optimal (polynomial) admissible mesh. It is related to particular results that have recently appeared in the literature for very special (globally semianalytic) sets like $N$-dimensional polynomial or analytic graph domains or polynomial and analytic polyhedrons. (Here a good source of references is the recent paper (Piazzon and Vianello, East J Approx 16(4):389-398, 2010).) We also show that an infinitely differentiable map $f$ from a compact set $Q$ in $\mathbb{R}^{N}$ onto a Markov compact set $K$ in $\mathbb{C}^{l}(l \leq N)$ transforms a (weakly) admissible mesh in $Q$ onto a (weakly) admissible mesh in $K$, which extends a result of Piazzon and Vianello (East J Approx 16(4):389-398, 2010) for analytic maps in case $Q$ is a subset of $\mathbb{R}^{N}$. Versions for $\mathcal{C}^{k}$ maps with sufficiently large $k$ are also given.
\end{abstract}

Keywords Admissible polynomial meshes • Optimal meshes • Subanalytic geometry $\cdot$ Hironaka rectilinearization theorem . Bernstein-Walsh-Siciak theorem • Jackson theorem

AMS 2000 Subject Classifications Primary 41A10; Secondary 32B20 • $32 \mathrm{U} 35 \cdot 41 \mathrm{~A} 17 \cdot 41 \mathrm{~A} 63 \cdot 65 \mathrm{D} 05$

Let $K$ be a compact subset of the $N$-dimensional complex space $\mathbb{C}^{N}$. Let $\mathbb{P}_{d}=$ $\mathbb{P}_{d}\left(\mathbb{C}^{N}\right)$ be the set of all polynomials on $\mathbb{C}^{N}$ of degree at most $d$ and let $\mathbb{P}=$

\footnotetext{
W. Pleśniak $(\bowtie)$

Wydział Matematyki i Informatyki, Uniwersytet Jagielloński, ul. Stanisława Łojasiewicza 6, 30-348 Kraków, Poland

e-mail: Wieslaw.Plesniak@im.uj.edu.pl
} 
$\bigcup_{d=1}^{\infty} \mathbb{P}_{d}$. A family $(A(d))_{d=1}^{\infty}$ of finite subsets $A(d)$ of $K$ is said to be a weakly admissible mesh if the cardinality of $A(d)$ grows polynomially when $d \rightarrow \infty$, i.e. $\# A(d)=O\left(d^{\alpha}\right)$, for some $\alpha>0$, and there exists a polynomially growing sequence $\{C(d)\}$ of positive constants such that for each $d \in \mathbb{N}$ and for all $P \in$ $\mathbb{P}_{d}$ one has

$$
\|P\|_{K} \leq C(A(d))\|P\|_{A(d)} .
$$

Here $\|h\|_{S}$ stands for the uniform norm sup $|h|(S)$. If moreover $\sup C(A(d))<$ $\infty$, then $(A(d))$ is said to be an admissible mesh. Suppose that $K$ is $\mathbb{P}$ determining, i.e. for each $P \in \mathbb{P}, P=0$ on $K$ forces $P(z) \equiv 0$. Then by the multivariate Langrange interpolation formula (see e.g. $[13,15])$ there is a weakly admissible mesh $(A(d))$ on $K$, where $A(d)$ is a set $\left\{t_{1}, \ldots, t_{m_{d}}\right\}$ of FeketeLeja type extremal points of $K$ of order $m_{d}:=\operatorname{dim} \mathbb{P}_{d}=\left(\begin{array}{c}N+d \\ N\end{array}\right)=\mathrm{O}\left(d^{N}\right)$. If $K$ is a Markov compact subset of $\mathbb{C}^{N}$, i.e. a compact set that admits a Markov inequality

$$
\|\nabla P\|_{K} \leq M d^{r}\|P\|_{K} \quad \text { for all } P \in \mathbb{P}_{d}
$$

with positive constants $M$ and $r$ depending only on $K$, then following [3] one can construct an admissible mesh $(A(d))$ on $K$ with $\# A(d)=O\left(d^{2 r N}\right)$ (and with $O\left(d^{r N}\right)$ cardinality, if $\left.K \subset \mathbb{R}^{N} \cong \mathbb{R}^{N}+i 0 \subset \mathbb{C}^{N}\right)$. Observe that $r \geq 1$ if $K \subset \mathbb{C}^{N}$ and $r \geq 2$ for any compact set $K \subset \mathbb{R}^{N}$ (cf also Example 7) and for computational reasons one would like to construct meshes with more modest cardinalities. On the other hand, for any $d \in \mathbb{N}, A(d)$ must be $\mathbb{P}_{d}$ - determining, whence $\# A(d) \geq m_{d}$. This leads to the notion of optimal polynomial meshes: an admissible mesh $(A(d))$ is said to be optimal, if \# $A(d)=O\left(d^{N}\right)$ as $d \rightarrow \infty$.

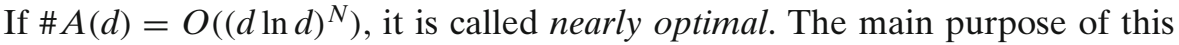
note is to show that nearly optimal meshes can be constructed on fat, compact subanalytic subsets of $\mathbb{R}^{N}$ that are known to admit Markov inequality (2) (see [8]). Let us first recall some basic notions of subanalytic geometry that was developed mainly by Łojasiewicz, Gabrielov and Hironaka.

A subset $E$ of $\mathbb{R}^{N}$ is said to be semianalytic if for each point $x \in \mathbb{R}^{N}$ one can find a neighbourhood $U$ of $x$ and a finite number of real analytic functions $f_{i j}$ and $g_{i j}$ defined in $U$, such that

$$
E \cap U=\bigcup_{i} \bigcap_{j}\left\{f_{i j}>0, g_{i j}=0\right\} .
$$

The projection of a semianalytic set need not be semianalytic ( $\mathrm{cf}[2,7])$. The class of sets obtained by enlarging that of semianalytic sets to include images under the projections has been called the class of subanalytic sets. More precisely, a subset $E$ of $\mathbb{R}^{N}$ is said to be subanalytic if for each point $x \in \mathbb{R}^{N}$ there exists an open neighbourhood $U$ of $x$ such that $E \cap U$ is the projection of a bounded semianalytic set $A$ in $\mathbb{R}^{N+M}$, where $M \geq 0$. If $N \geq 3$, the class of subanalytic sets is essentially larger than that of semianalytic sets, the classes being identical if $N \leq 2$. The union of a locally finite family and the 
intersection of a finite family of semianalytic (resp. subanalytic) sets is semianalytic (resp. subanalytic). The closure, interior, boundary and complement of a semianalytic (resp. subanalytic) set is still semianalytic (resp. subanalytic), the last property in the case of subanalytic sets being a (non-trivial) theorem of Gabrielov. For an excellent survey on subanalytic geometry, the reader is referred to [2]. In particular, one can find there an elegant proof of a crucial for this theory Hironaka Rectilinearization Theorem which (in a scalar space version) reads as follows.

Theorem 1 Let $E$ be a subanalytic subset of $\mathbb{R}^{N}$. Let $K$ be a compact subset of $\mathbb{R}^{N}$. Then there are finitely many real analytic mappings $\varphi_{j}: \mathbb{R}^{N} \mapsto \mathbb{R}^{N}$ such that:

(1) There is a compact subset $K_{j}$ of $\mathbb{R}^{N}$, for each j, such that $\bigcup_{j} \varphi_{j}\left(K_{j}\right)$ is a neighbourhood of $K$ in $\mathbb{R}^{N}$.

(2) $\varphi_{j}^{-1}(E)$ is a union of quadrants in $\mathbb{R}^{N}$.

With the aid of the above theorem one can prove (see [8]) the following

Theorem 2 Let $E$ be a bounded, subanalytic subset of $\mathbb{R}^{N}$ of pure dimension $N$. Then there are finitely many real analytic maps $f_{j}: \mathbb{R}^{N} \mapsto \mathbb{R}^{N}$ such that for each $j$,

$$
f_{j}\left(J^{N}\right) \subset E \quad \text { and } \bigcup_{j} f_{j}\left(I^{N}\right)=\bar{E} \text {, }
$$

where $J^{N}:=\left\{x \in \mathbb{R}^{N}:\left|x_{i}\right|<1, \quad i=1, \ldots, N\right\}$, and $I^{N}:=\left\{x \in \mathbb{R}^{N}:\left|x_{i}\right| \leq\right.$ $1, i=1, \ldots, N\}$.

Subanalytic geometry methods have appeared very useful in polynomial approximation, since they provide tools for investigating regularity of the pluricomplex Green's function (see e.g. [8, 9, 12, 13]). As an example, we refer the reader to an important application of Hironaka's theorem (in version of Theorem 2) which is the following

Corollary 3 [8] If $K$ is a fat (i.e. $K \subset \overline{\text { int } K}$ ) compact subanalytic subset of $\mathbb{R}^{N}$, then it admits Markov's inequality (2).

Actually, in [8], it has been shown essentially more, namely that the set $K$ of the above corollary is UPC, i.e. it is uniformly polynomially cuspidal and consequently, its pluricomplex Green function is Hölder continuous in $\mathbb{C}^{N}$.

We shall need a multidimensional version of the well-known BernsteinWalsh theorem which is due to Siciak [15]. 
Theorem 4 Let $K$ be a compact subset of the space $\mathbb{C}^{N}$. Assume that $K$ is polynomially convex, i.e. $K=\hat{K}:=\left\{z \in \mathbb{C}^{N}:|p(z)| \leq\|p\|_{K}\right.$ for all $\left.p \in \mathbb{P}\right\}$. If $f$ is a holomorphic function in an open neighbourhood of $K$ then

$$
\limsup _{n \rightarrow \infty} \sqrt[n]{\operatorname{dist}_{K}\left(f, \mathbb{P}_{n}\right)}<1 .
$$

One can also easily prove the following

Lemma 5 (cf [13]) If $K$ is a Markov compact set in $\mathbb{C}^{N}$ then for every polynomial $P \in \mathbb{P}_{d}(d=1,2 \ldots)$,

$$
|P(z)| \leq e^{N}\|P\|_{K} \quad \text { if } \operatorname{dist}(z, K) \leq \frac{1}{M d^{r}},
$$

where $M$ and $r$ are the constants of inequality (2).

Now we can state the main result of this paper.

Theorem 6 Let $K$ be a fat, compact subanalytic subset of $\mathbb{R}^{N}$. Then one can construct an admissible mesh $(A(d))$ on $K$ such that $\# A(d)=O\left((d \ln d)^{N}\right)$ as $d \rightarrow \infty$.

Proof Let

$$
f_{j}=\left(f_{j, 1}, \ldots, f_{j, N}\right): \mathbb{R}^{N} \mapsto \mathbb{R}^{N}(j=1, \ldots, m)
$$

be real analytic functions of Theorem 2 for $\bar{E}=K$. Let $P \in \mathbb{P}_{d}$. Choose a point $w \in K$ such that $|P(w)|=\|P\|_{K}$. Then there is $j \in\{1, \ldots, m\}$ such that $w \in f_{j}(I)$. Now choose $x \in I$ such that $w=f_{j}(x)$. Since any compact set in $\mathbb{R}^{N}$ is polynomially convex, by Theorem 4 there exist polynomials $P_{n, k} \in \mathbb{P}_{n}$, $n=1,2, \ldots$, and constants $L>0$ and $a \in(0,1)$ independent of $n$ such that

$$
\left\|f_{j, k}-P_{n, k}\right\| \leq L a^{n}=: \varepsilon_{n}
$$

for $k=1, \ldots, N$. Set $P_{n}=\left(P_{n, 1}, \ldots, P_{n, N}\right)$. Let $w^{n}=P_{n}(x)$. Then $\| w-$ $w^{n}\|=\| f_{j}(x)-P_{n}(x) \| \leq \sqrt{N} \varepsilon_{n}$. Let $(A(d))_{d=1}^{\infty}$ be an optimal admissible mesh in the cube $I$. (It is well-known that such meshes exist; e.g. one can take the Cartesian product of a one dimensional mesh $Y(d)$ on $[-1,1]$ with $\# Y(d)=$ $O(d)$, constructed in [4], chap. 3, sec.7, Lemma 3.) By the mean value theorem, Lemma 5 and Markov's inequality (2), we have

$$
\left|P(w)-P\left(w^{n}\right)\right| \leq\|\nabla P\|_{\left[w, w^{n}\right]}\left\|w-w^{n}\right\| \leq N e^{N} M d^{r}\|P\|_{K} \varepsilon_{n},
$$

provided $\sqrt{N} \varepsilon_{n} \leq \frac{1}{M d^{r}}$. Hence, setting $\varphi(d, n):=N e^{N} M d^{r} \varepsilon_{n}$ gives

$$
\begin{aligned}
\|P\|_{K} & =|P(w)| \leq\left|P(w)-P\left(w^{n}\right)\right|+\left|P\left(w^{n}\right)\right| \\
& \leq \varphi(d, n)\|P\|_{K}+C\|P\|_{P_{n}(A(d n))}
\end{aligned}
$$


with $C=C(A(d)) \geq 1$, as $\sqrt{N} \varepsilon_{n} \leq 1 / M d^{r}$. By a similar way, we shall now estimate $\|P\|_{P_{n}(A(d n))}$. Let $z \in P_{n}(A(d n))$ be such that $|P(z)|=\|P\|_{P_{n}(A(d n))}$. Choose $y \in A(d n)$ so that $P_{n}(y)=z$. We have

$$
\begin{aligned}
|P(z)| & \leq\left|P\left(P_{n}(y)\right)-P\left(f_{j}(y)\right)\right|+\left|P\left(f_{j}(y)\right)\right| \\
& \leq \varphi(d, n)\|P\|_{K}+C\|P\|_{f_{j}(A(d n))} .
\end{aligned}
$$

Hence by (5),

$$
\|P\|_{K} \leq \varphi(d, n)\|P\|_{K}+C \varphi(d, n)\|P\|_{K}+C^{2}\|P\|_{A^{\prime}(d n)},
$$

where $A^{\prime}(d n):=\bigcup_{j=1}^{m} f_{j}(A(d n))$, provided $\sqrt{N} \varepsilon_{n} \leq 1 / M d^{r}$. Now, it is easily seen that there is a sequence $n(d)=O(\ln d)$ of positive integers such that $\varphi(d, n(d)) \leq \frac{C}{4}$ and $\sqrt{N} \varepsilon_{n} \leq 1 / M d^{r}$. Then

$$
\|P\|_{K} \leq 2 C^{2}\|P\|_{A^{\prime}(\operatorname{dn}(d))} .
$$

One also verifies that \# $A^{\prime}(d n(d))=O\left((d \ln d)^{N}\right)$.

In general, Theorem 6 gives better estimates of the cardinality of accessible meshes in subanalytic sets than those yielded by [3, Theorem 5]. This is seen by the following

Example 7 Consider the set

$$
K=\left\{x=\left(x_{1}, x_{2}\right) \in \mathbb{R}^{2}: 0 \leq x_{1} \leq 1,0 \leq x_{2} \leq g\left(x_{1}\right)\right\},
$$

where $g$ is an analytic function in an open neighbourhood of [0,1] such that $0<g\left(x_{1}\right) \leq x_{1}^{p}$ for some $p \in \mathbb{N}$. Then $K$ is a semianalytic set, whence by Corollary 3 it is Markov. Its Markov exponent $r$ has to be greater than $M \frac{p}{\ln p}$ for $p$ sufficiently large, which can be easily seen by considering the polynomials $P\left(x_{1}, x_{2}\right)=x_{2}\left(1-x_{1}\right)^{p}$. (Actually, if $g\left(x_{1}\right)=x_{1}^{p}$, then by Goetgheluck [5] $r=$ $2 p$.) Thus Markov's exponent of $K$ could be as large as we want. By Theorem 6 one can construct an admissible mesh $(A(d))$ in $K$ with $\# A(d)=O\left((d \ln d)^{2}\right)$, as $d \rightarrow \infty$, while by [3, Theorem 5] we know only that there exists an admissible mesh $(A(d))$ in $K$ with $\# A(d)=O\left(d^{2 r}\right)$.

The idea of applying Markov's inequality and the mean value theorem to constructing admissible meshes goes back to Cheney and it has been described in his monograph [4] in the case of univariate polynomial approximation. In the proof of the above theorem we also exploit the possibility of rapid (geometric) approximation of analytic maps by polynomials. Such a method has also been used by the authors of the recent interesting paper [10], where they prove the following

Theorem 8 Let $K$ be a Markov compact subset of $\mathbb{C}^{N}$ and let $Q$ be a $\mathbb{P}$ determining compact set in $\mathbb{C}^{N}$ such that $K=f(Q)$, where $f$ is an analytic map in an open neighbourhood of the polynomial hull $\hat{Q}$ of $Q$. Let $(A(d))$ be a 
(weakly) admissible mesh for $Q$. Then there exists a sequence $j(d)=O(\ln d)$ of natural numbers such that $\left(A^{\prime}(d)\right):=((f(A(d j(d))))$ is a (weakly) admissible mesh for $K$ with $C\left(A^{\prime}(d)\right) \asymp C(A(d j(d)))$ and \# $A^{\prime}(d) \leq \# A(d j(d))$.

Observe that in the above theorem we are able to let $f$ have values in the space $\mathbb{C}^{l}$ with $l \leq N$. Let us also note that we cannot directly apply Theorem 8 in the proof of Theorem 6 , since we do not know whether the sets $f_{j}(I)$ are Markov. We only know, by [1], that this is the case if $\operatorname{det}\left[f_{j}^{\prime}(x)\right] \neq 0$ at every point $x \in I$.

Remark 9 In a recent paper [6], Kroó constructs admissible meshes in graph domains in $\mathbb{R}^{N}$ that are sets of the type

$$
\begin{aligned}
K_{g}:= & \left\{\left(x_{1}, \ldots, x_{N}\right) \in \mathbb{R}^{N}: f_{k}\left(x_{1}, \ldots, x_{k-1}\right) \leq x_{k} \leq g_{k}\left(x_{1}, \ldots, x_{k-1}\right),\right. \\
& \left.\left(x_{1}, \ldots, x_{k-1}\right) \in I^{k-1}, 1 \leq k \leq N\right\},
\end{aligned}
$$

where $I^{k}=[0,1]^{k}, 1 \leq k \leq N, f_{1} \equiv 0, g_{1} \equiv 1$ and $0 \leq f_{k}(x) \leq g_{k}(x) \leq 1, x \in$ $I^{k-1}, 2 \leq k \leq N$. (Such domains are also called "normal domains" in textbooks on multiple integrals.) He shows (Proposition 1) that in case the functions $f_{k}$ and $g_{k}$ are algebraic polynomials the domain $K_{g}$ possesses an optimal polynomial mesh. Actually, it immediately follows from the fact that any graph set $K_{g}$ is simply the image of the cube $[0,1]^{N}$ by the map

$$
\begin{aligned}
F\left(t_{1}, \ldots, t_{N}\right):= & \left(t_{1},\left(1-t_{2}\right) f_{2}\left(t_{1}\right)+t_{2} g_{2}\left(t_{1}\right), \ldots,\right. \\
& \left.\left(1-t_{N}\right) f_{N}\left(t_{1}, \ldots, t_{N-1}\right)+t_{N} g_{N}\left(t_{1}, \ldots, t_{N-1}\right)\right) .
\end{aligned}
$$

Indeed, if $(A(d))$ is an optimal mesh in $I^{N}$ and $F=\left(F_{1}, \ldots, F_{N}\right): \mathbb{R}^{N} \mapsto \mathbb{R}^{N}$ is a polynomial map of degree $s=\max _{1 \leq k \leq N} \operatorname{deg} F_{k}$, then for any polynomial $P$ in $\mathbb{R}^{N}$ of degree $d$ one has

$$
\|P\|_{F\left(I^{N}\right)}=\|P \circ F\|_{I^{N}} \leq C\|P \circ F\|_{A(s d)} \leq C\|P\|_{F(A(s d))}
$$

with \#F( $A(s d)) \leq \# A(s d) \leq M s^{N} d^{N}$. The same holds true if $K$ is a finite union of the images $F^{j}\left(I^{N}\right)$ of the unit cube $I^{N}$ by polynomial maps $F^{j}: \mathbb{R}^{N} \mapsto \mathbb{R}^{N}$, in particular if $K$ is a polytope.

If the functions $f_{k}$ and $g_{k}$ are traces on $I^{k-1}$ of real analytic functions then the corresponding graph domain $K_{g}$ is clearly a (global) semianalytic set. Then by Theorem 6 one can construct in $K_{g}$ an admissible mesh $(A(d))$ with $\# A(d)=O\left((d \ln d)^{N}\right)$ which is better than the estimate \#A(d)= $O\left(d^{N} \ln ^{N(N-1)} d\right)$ yielded in such a case by [6, Theorem 1]. Let us add that in the analytic case the cardinality result $\# A^{\prime}(d)=O\left((d \ln d)^{N}\right)$ for $K_{g}$ also follows from Corollary 3 and Theorem 7.

Other typical sets fulfilling the assumptions of Theorem 6 are analytic polyhedrons, i.e. compact subsets $K$ of a domain $\Omega$ in $\mathbb{R}^{N}$ of the type

$$
K:=\left\{x \in \Omega:\left|h_{j}(x)\right| \leq 1, j=1, \ldots, m\right\},
$$

where $h_{j}$ are real analytic functions in $\Omega$. 
Now we are going to show that in case $Q$ is a subset of $\mathbb{R}^{N}$ Theorem 8 is also valid for $\mathcal{C}^{\infty}$ maps and even for $\mathcal{C}^{k}$ maps with sufficiently large $k$ depending on Markov's exponent $r$ of inequality (2) and the growth of the sequence $\{C(A(d))\}$.

Theorem 10 Let $Q$ be a compact set in $\mathbb{R}^{N}$ and let $f=\left(f_{1}, \ldots, f_{l}\right)$ be a map defined on $Q$, with values in $\mathbb{C}^{l}(l \leq N)$, whose components $f_{j}$ are traces of $\mathcal{C}^{\infty}$-functions on $\mathbb{R}^{N}$. Suppose that the set $K=f(Q)$ is Markov. Let $(A(d))$ be a (weakly) admissible mesh in $Q$. Then there is a positive integer $m$ such that $\left(f\left(A\left(m d^{2}\right)\right)\right)$ is a (weakly) admissible mesh in $K$.

Proof By the multivariate Jackson theorem (applied to a cube $I \supset Q$ in $\mathbb{R}^{N}$ ), one can find polynomials $P_{j, n} \in \mathbb{P}_{n}$ such that the sequence $\varepsilon_{j, n}:=\| f_{j}-$ $P_{j, n} \|_{Q}$ is rapidly decreasing, i.e. for each $k>0, n^{k} \varepsilon_{j, n} \rightarrow 0$ as $n \rightarrow \infty$ for $j=1, \ldots, l($ see $[13,16])$. Let $P_{n}=\left(P_{1, n}, \ldots, P_{l, n}\right)$ and $\varepsilon_{n}=\max _{j} \varepsilon_{j, n}$. We have $\left\|f-P_{n}\right\|_{Q} \leq \sqrt{l} \varepsilon_{n}$. Take a polynomial $W \in \mathbb{P}_{d}\left(\mathbb{C}^{l}\right)$ and choose $w \in K=f(Q)$ so that $|W(w)|=\|W\|_{K}$. Then, by a similar argument to that of the proof of Theorem 6 (cf also the proof of Theorem 7 in [10]) we arrive at the estimate

$$
\begin{aligned}
\|W\|_{K} \leq & \psi(d, n)\|W\|_{K}+C(A(d n)) \psi(d, n)\|W\|_{K} \\
& +C(A(d n))\|W\|_{f(A(d n))}
\end{aligned}
$$

with $\psi(d, n):=M l e^{l} d^{r} \varepsilon_{n}$, provided $\sqrt{l} \varepsilon_{n} \leq 1 / M d^{r}$. Observe that for each $k>0$ we have

$$
\psi(d, n)=\text { Const. } n^{k} \varepsilon_{n} \frac{d^{r}}{n^{k}} \leq \text { Const. } \sup _{n}\left(n^{k} \varepsilon_{n}\right) \frac{d^{r}}{n^{k}}=C(k) \frac{d^{r}}{n^{k}} .
$$

Consider now two cases.

$1^{\circ} \quad C:=\sup _{d} C(A(d))<\infty$, that is the mesh $(A(d))$ is admissible. We may assume that $C \geq 1$. Then, setting $k=[r]+1$, where $[r]$ denotes the entire part of $r$, one can find a positive integer $m$ such that $C \psi(d, m d) \leq \frac{1}{4}$ and $\varepsilon_{m d} \leq 1 / M d^{r}$. Consequently,

$$
\|W\|_{K} \leq 2 C\|W\|_{f\left(A\left(m d^{2}\right)\right.},
$$

and if $\# A(d)=O\left(d^{\alpha}\right)$ for some $\alpha>0$, we get \# $f\left(A\left(m d^{2}\right)=O\left(d^{2 \alpha}\right)\right.$. Thus $\left(f\left(A\left(m d^{2}\right)\right)\right)$ is an admissible mesh in $K$.

$2^{\circ} \quad$ Suppose $C(A(d))=O\left(d^{\beta}\right)$ for some $\beta>0$. Then again, setting $k=[\beta+$ $r]+1$, we can find a positive integer $m^{\prime}$ such that $C\left(A\left(m^{\prime} d^{2}\right)\right) \psi\left(d, m^{\prime} d\right) \leq$ $\frac{1}{4}$ and $\varepsilon_{m d} \leq 1 / M d^{r}$. This yields the inequality

$$
\|W\|_{K} \leq 2 C\left(A\left(m^{\prime} d^{2}\right)\right)\|W\|_{f\left(a\left(m^{\prime} d^{2}\right)\right)} .
$$




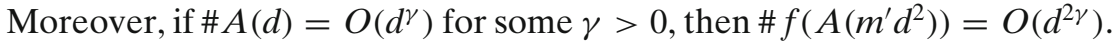
This means that the mesh $\left(f\left(A\left(m^{\prime} d^{2}\right)\right)\right)$ is weakly admissible.

Remark 11 By a version of the multivariate Jackson theorem in [16], if a map $f=\left(f_{1}, \ldots, f_{l}\right)$ defined on $Q$ extends to a $\mathcal{C}^{k+1}$ map from $\mathbb{R}^{N}$ to $\mathbb{C}^{l}$, then for each $j \in\{1, \ldots, l\}$,

$$
\sup _{n} n^{k} \varepsilon_{n} \leq C(k) \sum_{|\alpha| \leq k+1}\left\|D^{\alpha} f_{j}\right\|_{I} \leq D(k, f),
$$

where $I$ is a compact cube in $\mathbb{R}^{N}$ containing the set $Q$. Then, if the mesh $(A(d))$ is admissible, Theorem 10 holds if $f$ is a $\mathcal{C}^{[r]+2}$ map, and if $C(A(d))=O\left(d^{\beta}\right)$ $(\beta>0)$, then Theorem 10 is valid for any $\mathcal{C}^{[\beta+r]+2}$ map $f$.

Remark 12 By a non-trivial result of [11], bounded, fat and definable sets in some polynomially bounded o-minimal structures generated by special classes of $C^{\infty}$ functions in $\mathbb{R}^{N}$ are uniformly polynomially cuspidal, whence by [8] they are Markov. This is e.g. the case of the Rolin-Speissegger-Wilkie structure (cf [14]) generated by the Denjoy-Carleman classes of quasianalytic functions with partial derivatives tempered by a strongly logarithmically convex sequence $\left\{M_{p}\right\}$. In [11], Pierzchała has proved a version of Theorem 2 for such a structure. Thus it should be possible to extend Theorem 6 to the case of definable sets in the Rolin-Speissegger-Wilkie o-minimal structure.

Open Access This article is distributed under the terms of the Creative Commons Attribution License which permits any use, distribution, and reproduction in any medium, provided the original author(s) and the source are credited.

\section{References}

1. Baran, M., Pleśniak, W.: Markov's exponent of compact sets in $\mathbb{C}^{n}$. Proc. Am. Math. Soc. 123, 2785-2791 (1995)

2. Bierstone, E., Milman, P.D.: Semianalytic and subanalytic sets. Publ. Math. Inst. Hautes. Études Sci. 67, 5-42 (1988)

3. Calvi, J.-P., Levenberg, N.: Uniform approximation by discrete least squares polynomials. J. Approx. Theory 152, 82-100 (2008)

4. Cheney, E.W.: Introduction to Approximation Theory. AMS Chelsea Publishing, Providence, Rhode Island (1982)

5. Goetgheluck, P.: Inégalité de Markov dans les ensembles effilés. J. Approx. Theory 30,149154 (1980)

6. Kroó, A.: On optimal polynomial meshes. J. Approx. Theory 163, 1107-1124 (2011)

7. Łojasiewicz, S.: Ensembles semi-analytiques. Inst. Hautes Études Sci., Bures-sur-Yvette (1964)

8. Pawłucki, W., Pleśniak, W.: Markov's inequality and $\mathcal{C}^{\infty}$ functions on sets with polynomial cusps. Math. Ann. 275, 467-480 (1986)

9. Pawłucki, W., Pleśniak, W.: Extension of $C^{\infty}$ functions from sets with polynomial cusps. Studia Math. 88(3), 279-287 (1988)

10. Piazzon, F., Vianello, M.: Analytic transformations of admissible meshes. East J. Approx. 16(4), 389-398 (2010) 
11. Pierzchała, R.: UPC condition in polynomially bounded o-minimal structures. J. Approx. Theory 132, 25-33 (2005)

12. Pleśniak, W.: L-regularity of subanalytic sets in $\mathbb{R}^{n}$. Bull. Acad. Polon. Sci. Sér. Sci. Math. 32, 647-651 (1984)

13. Pleśniak, W.: Markov's inequality and the existence of an extension operator for $\mathcal{C}^{\infty}$ functions. J. Approx. Theory 61, 106-117 (1990)

14. Rolin, J.-P., Speissegger, P., Wilkie, A.J.: Quasianalutic Denjoy-Carleman classes and o-minimality. J. Am. Math. Soc. 16(4), 751-777 (2003)

15. Siciak, J.: On some extremal functions and their applications in the theory of analytic functions of several complex variables. Trans. Am. Math. Soc. 105, 322-357 (1962)

16. Timan, A.F.: Theory of Approximation of Functions of a Real Variable. Oxford Pergamon Press (1963) 